\title{
Probing the Electromagnetic Field Distribution within a Metallic Nanodisk
}

David Meneses-Rodríguez, Elías Ferreiro-Vila, Patricia Prieto, José Anguita, María U. González, José M. García-Martín, Alfonso Cebollada, Antonio García-Martín, and Gaspar Armelles*

[*] Prof. G. Armelles

IMM-Instituto de Microelectrónica de Madrid (CNM-CSIC)

Isaac Newton 8, PTM, E-28760 Tres Cantos, Madrid, SPAIN.

Fax: (+34)918 060701

E-mail: gaspar@imm.cnm.csic.es

Keywords: Magneto-Optical Activity, Plasmonics, Localized Surface Plasmon Resonance, Nanodisk.

A Co nanolayer is used as a local probe of the vertical inhomogeneous distribution of the electromagnetic (EM) field within a metallic resonant nanodisk. Taking advantage of the direct relation between the magnetooptical activity and the electromagnetic field intensity at the Co layer, we show the EM non-uniform distribution within the nanodisks in plasmon resonance conditions, with maximum values close to the upper and lower flat faces, and a minimum value in the middle.

\section{Introduction}

Plasmonic resonances in metallic nanostructures have attracted a great deal of interest due to their wide range of applications ${ }^{[1]}$, including waveguides $^{[2,3]}, \operatorname{microscopies}^{[4,5,6,7]}$, $\operatorname{lasers}^{[8,9]}$, surface-enhanced Raman scattering (SERS) $)^{[10,11]}$ and biological sensors ${ }^{[12,13,14,15]}$. These photon-electron excitations allow confining the electromagnetic waves to nanoscale dimensions resulting in strong field enhancement ${ }^{[16]}$, and are very sensitive to the optical properties of their environment. Most of these applications, such as SERS, sensing or the design of nanoplasmonic devices, rely on the EM field localization and are therefore strongly determined by its distribution. 
Near field optical techniques allow determining experimentally the EM field distribution in the close vicinity of the structures ${ }^{[17,18]}$. Techniques based on electron microscopy, such as electron energy-loss spectroscopy $(E E L S)^{[19]}$ or photoelectron emission microscopy $(\mathrm{PEEM})^{[20]}$, provide a planar view, real-space imaging of the plasmonic resonance with a resolution of a few tens of nanometers, integrating the information in the vertical section. Unfortunately, it is not straightforward to extract similar information from the region inside a metallic structure and along the vertical axis. Moreover, this EM field distribution becomes greatly modified and asymmetric in the presence of a substrate. For instance, in figure 1(a) we show the calculated EM field distribution within a Au nanodisk of $130 \mathrm{~nm}$ diameter and 54 $\mathrm{nm}$ height placed over a glass substrate upon plane wave illumination at normal incidence when the localized surface plasmon resonance (LSPR) is excited. We can see that the EM field is maximum at both interfaces with a minimum at the center of the disk. This is in strong contrast with the continuous decay presented by a thin continuous metallic film deposited on a dielectric substrate, where the EM field is maximum at the air-metal interface, decaying exponentially within the metal and, less abruptly, within the substrate.

However, and despite its obvious interest, the EM field distribution inside metallic resonant nanodisks has not been experimentally available so far. Fortunately, it is possible to obtain this information indirectly, by measuring a far field quantity that depends on the field inside the nanostructure. Our approach lies in the influence that the EM field inside the metallic disk has on the magnetooptical (MO) activity of a very thin Co layer, that can be placed at will in different positions along the vertical direction within this disk.

Recently, the incorporation of magnetic materials into plasmonic structures to constitute the so-called magnetoplasmonic systems has allowed obtaining active plasmonic entities. For example, it is possible to modulate the surface plasmon wavevector in $\mathrm{Au} / \mathrm{Co} / \mathrm{Au}$ continuous films by the application of an external magnetic field ${ }^{[21,22]}$. Actually, measuring this modulation as a function of the Co layer position in the structure or in the presence of a 
dielectric overlayer, it is possible to probe the EM field distribution within a continuous opaque metallic film ${ }^{[21,22]}$. In this work, we have studied the $\mathrm{MO}$ activity of $\mathrm{Au} / \mathrm{Co} / \mathrm{Au}$ nanodisks as a function of the position of the Co layer along the vertical direction as a way to experimentally probe the EM field distribution within the nanodisk in the case of LSPR excitation. This layer can be located at different spatial locations within the disk to act as an EM field probe, since the MO activity is proportional to the EM field at the MO active layer[ $[1,22,23,24,25,26,27,28]$.
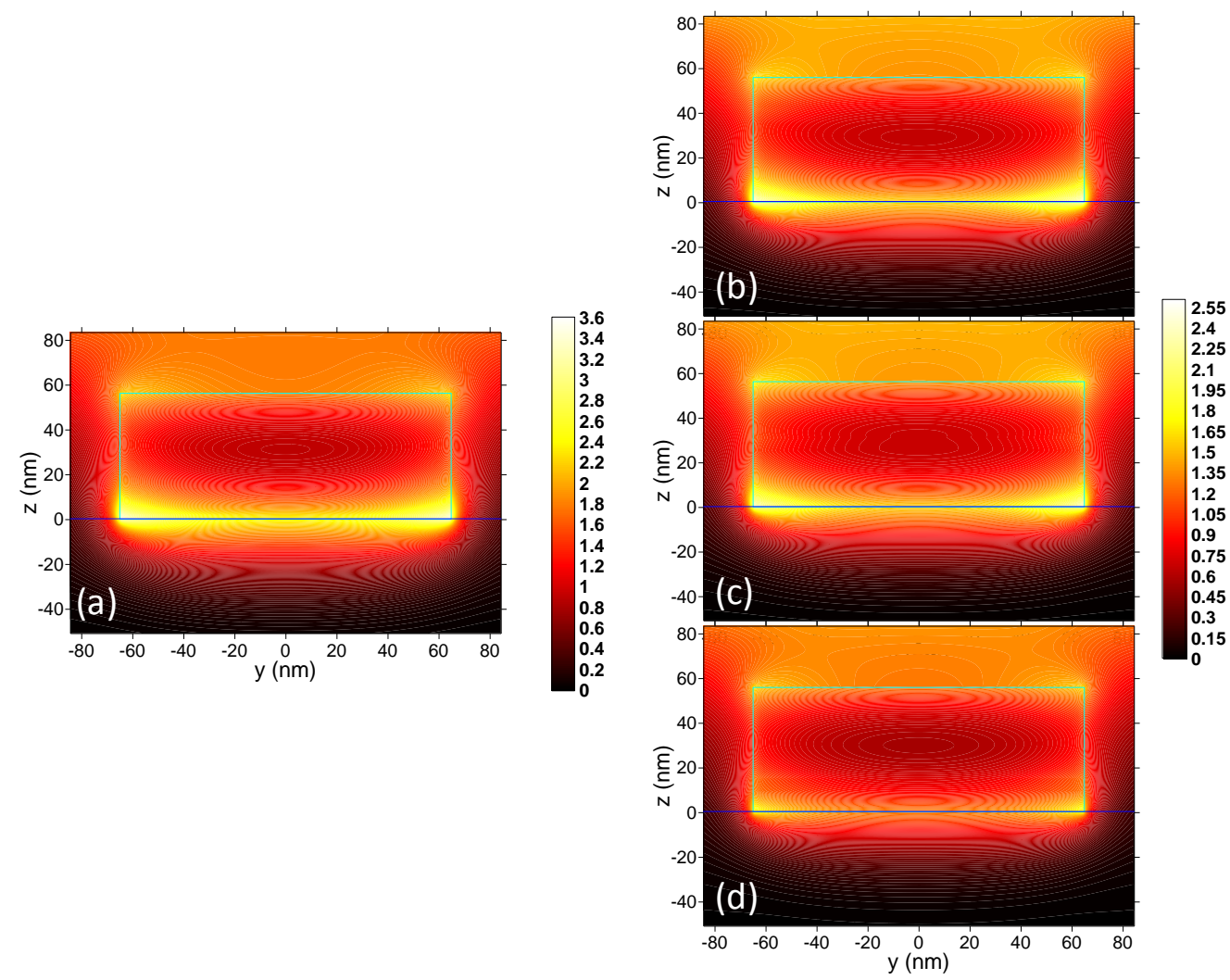

Figure 1: EM field distribution within a metallic nanodisk. EM field distribution calculated using a finite-difference time-domain (FDTD) technique, at LSPR excitation, within: (a) a $130 \mathrm{~nm}$ diameter and $54 \mathrm{~nm}$ height Au nanodisk when a localized surface plasmon resonance (LSPR) is excited; same Au nanodisk with a thin $(6 \mathrm{~nm})$ Co layer placed (b) close to the upper interface, (c) in the middle and (c) close to the lower interface of the nanodisk respectively. 


\section{Results and Discussion}

A crucial issue is to what extent this Co probe induces itself a perturbation in the EM field distribution within the nanodisk. We have first calculated the effect produced by a $6 \mathrm{~nm}$ thick Co layer placed within the Au nanodisk of $130 \mathrm{~nm}$ diameter and $54 \mathrm{~nm}$ height shown in Figure 1(a). Figures 1(b)-(d) show the EM field distributions for three cases: with the Co layer placed close to the upper interface (Figure 1(b)), in the middle of the nanodisk (Figure 1(c)) and close to the lower interface (Figure 1(d)) respectively. When compared with the pure Au disk (figure 1(a)), it can be clearly seen that the insertion of such a thin Co layer has very little influence in the shape of the EM field distribution except for a mere overall decrease of the intensity due to the larger absorption of Co. The effect is analogous irrespective of the Co layer position within the disk, and confirms the almost non-perturbative nature of this Co layer as an EM field probe.

The $\mathrm{Au} / \mathrm{Co} / \mathrm{Au}$ nanodisks samples were fabricated by means of colloidal lithography ${ }^{[29,30,31,32],}$ and sputter deposition (see experimental section). All samples have the same nanodisks diameter $(130 \mathrm{~nm})$, height $(54 \mathrm{~nm})$, and density $\left(9.4 \mu \mathrm{m}^{-2}\right)$. Each individual disk has a four layer structure: a $2 \mathrm{~nm}$ thick Cr layer to improve the adhesion to the substrate; a first Au layer, whose thickness d1 varies from sample to sample between 5 and $35 \mathrm{~nm}$; a $6 \mathrm{~nm}$ thick Co layer; and a second Au layer whose thickness $d_{2}$ also varies from sample to sample to maintain the total Au thickness $\left(d_{1}+d_{2}\right)$ equal to $46 \mathrm{~nm}$. This way, by varying $\mathrm{d} 1$, the position of the Co probe layer within the structure is also varied. In order to compare with identical $\mathrm{Cr} / \mathrm{Au} / \mathrm{Co} / \mathrm{Au}$ multilayered continuous films, an area of each substrate was left uncovered of resist for simultaneous deposition of a continuous reference film.

The structures fabricated in this way are sketched in Figure 2(a), both in nanodisk (left) and continuous film form (right), where the parameter " $\mathrm{z}$ " represents the distance to the substrate surface of the central part of the Co layer. In Figure 2(b) a representative SEM image of one of the samples is presented, showing an in-plane random distribution. The average coverage, 
obtained from both AFM and SEM characterization, is $12.5 \%$, which corresponds to an average interdisk distance of $350 \mathrm{~nm}$ and makes the interdisk interaction effects negligible.

(a)
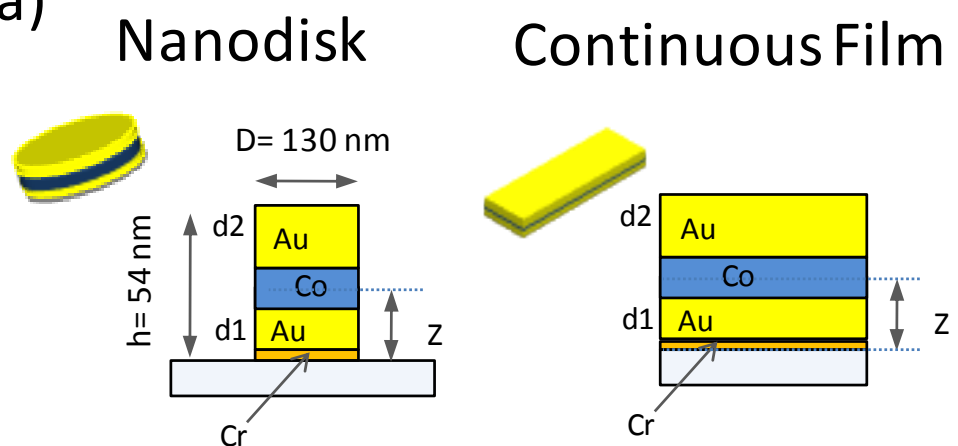

(b)

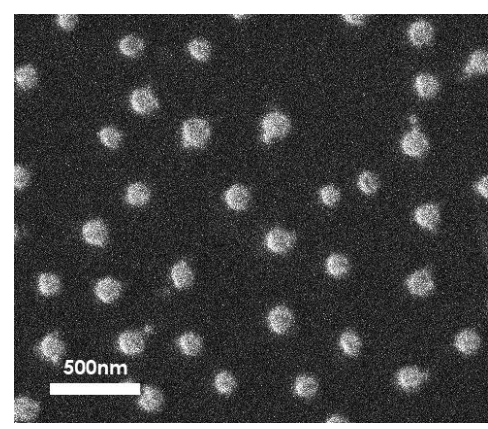

(c)

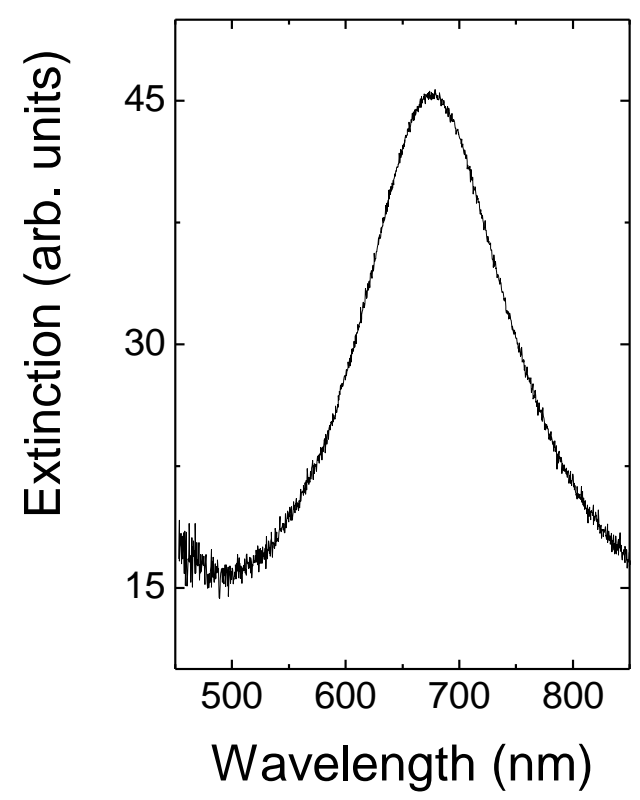

Figure 2: Structure and morphology of the fabricated samples. (a) Sketch of the fabricated samples; (b) SEM image of a representative structure; (c) extinction spectrum of the sample shown in (b). The extinction peak is slightly broader than the corresponding to a pure Au disc due to the absorption of the Co layer. 
The extinction spectrum of the same sample is presented in the figure 2(c). As it can be seen, a clear resonance peak is observed around $670 \mathrm{~nm}$, corresponding to the LSPR of the nanodisks.

The MO characterization of the fabricated structures has been carried out by means of Kerr spectroscopy in the polar configuration ${ }^{[33]}$. In figure 3(a) we present the Kerr rotation $(\theta)$ and ellipticity $(\varphi)$ spectra for three representative $\mathrm{Au} / \mathrm{Co} / \mathrm{Au}$ structures, with the Co layer close to the top interface, in the middle of the disc and close to the bottom interface. As it can be observed, in the spectral region of the nanodisk LSPR both the Kerr rotation and ellipticity spectra present specific features with different shapes: the Kerr rotation feature has an S-like shape, whereas the ellipticity spectra present a peak. This wavelength dependence is the same for all the different $\mathrm{Au} / \mathrm{Co} / \mathrm{Au}$ nanodisks samples. In figure 3(b) we present the corresponding modulus $\left(|\Phi|=\sqrt{\theta^{2}+\varphi^{2}}\right)$ of the complex Kerr rotation, $\Phi(\Phi=\theta+i \varphi)$ for the same three samples. As it can be seen, this modulus exhibits a clear resonance peak in the $670 \mathrm{~nm}$ region for the three cases, which is the same spectral region where the extinction peak is observed.

Identical behavior is obtained in all the fabricated nanodisks samples, and is well known to be related to the magnetooptical activity enhancement associated with the LSPR excitation, as previously shown in a number of magneto-plasmonic nanoentities ${ }^{[34,35,36,37,38,39,40,41]}$. Notably, here we find that the $\Phi$ peak intensity strongly depends on the position of the Co layer, being higher for the sample where the Co is located in the lower part of the nanodisk, closer to the glass substrate, with less intensity for the sample where the Co is located in the upper part of the nanodisc, and with a minimum magnetooptical activity for the sample with the Co in the middle of the nanodisc. This behavior is not the one expected for $\mathrm{Au} / \mathrm{Co} / \mathrm{Au}$ multilayered continuous films. Figure 3(c) shows the modulus of the complex Kerr rotation for the continuous films with multilayer sequences equivalent to those of the nanodisks shown in figure 3(b). In these cases, and for all the continuous films regardless of the Co layer position, 
the modulus of the complex Kerr rotation shows a peak at $500 \mathrm{~nm}$ arising from the Au plasma edge $^{[24]}$. The intensity of this peak also depends on the position of the Co layer but, in strong contrast with the $\mathrm{Au} / \mathrm{Co} / \mathrm{Au}$ nanodisk samples, it gradually decreases as the Co layer moves from the upper to the lower interface.

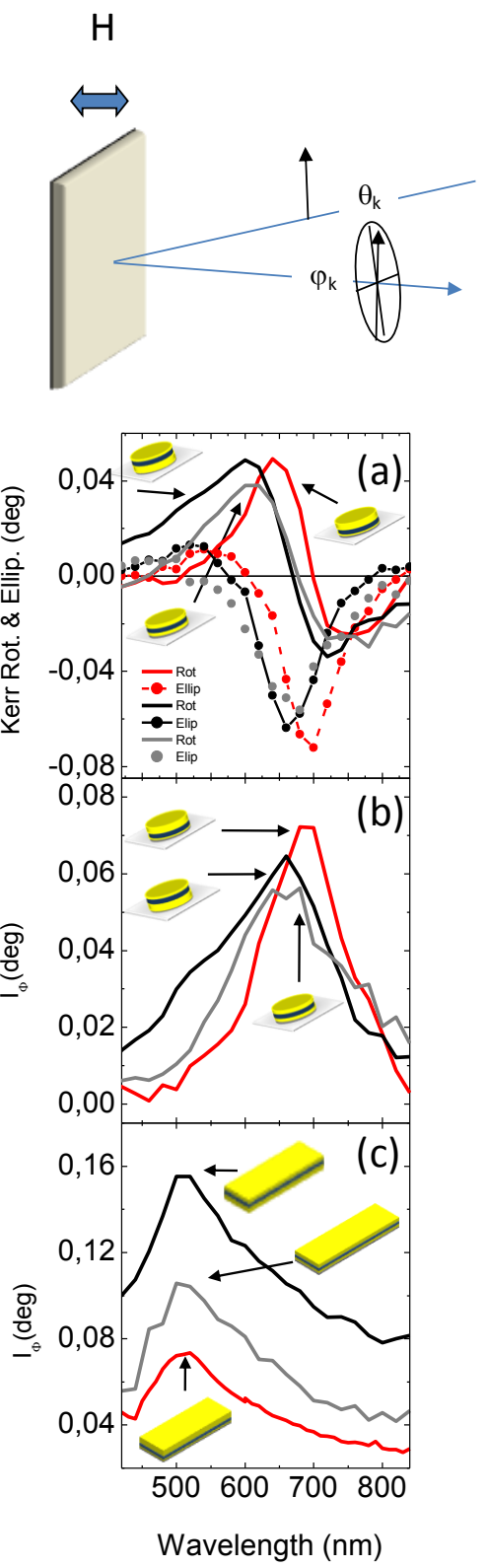

Figure 3: Magneto-optic characterization. (Upper inset) Sketch of the Polar Kerr measurement. (a) Polar Kerr rotation spectra (full lines) and ellipticity (dotted lines) of three different nanodisks samples (red lines, $\mathrm{z}=12 \mathrm{~nm}$; grey lines, $\mathrm{z}=28 \mathrm{~nm}$ and black lines, $\mathrm{z}=39$ $\mathrm{nm}$ ). (b) Modulus of the complex Kerr rotation spectra of the nanodisks samples shown in (a). (c) Modulus of the complex Kerr rotation spectra for the equivalent continuous films. 
This different behavior of the dependence of the MO activity with the Co layer position for both structures is better seen in figure 4 . Here it is shown the $\Phi$ peak intensity as a function of the distance of the Co layer to the bottom interface, z (see figure 2) for both films (a) and $\mathrm{Au} / \mathrm{Co} / \mathrm{Au}$ nanodisks (b). Two clearly distinctive behaviors are observed. In the case of the continuous films, the intensity gradually decreases as the Co layer is placed further from the upper interface. For the disks, on the other hand, the intensity shows a U-like shape, with maximum values when the Co layer is near the bottom (glass/Au) and upper (Au/air) interfaces, and lower ones at intermediate positions. A way to understand this different behavior is to analyze the system in a thin film approximation (i.e. assume that the Co layer is very thin so that it does not perturb the EM distribution). Let us remind that for a ferromagnetic material magnetically saturated along the $\mathrm{z}$ direction, the dielectric tensor can be expressed as:

$$
\left(\begin{array}{ccc}
\varepsilon & \varepsilon_{M O} & 0 \\
-\varepsilon_{M O} & \varepsilon & 0 \\
0 & 0 & \varepsilon
\end{array}\right)
$$

For $\mathrm{Co}$, the off diagonal component, $\varepsilon_{\mathrm{MO}}$, is smaller than the diagonal component, $\varepsilon$, and the modulus of the complex Kerr rotation is proportional to the modulus of $\varepsilon_{\mathrm{MO}}$ :

$$
|\Phi|=\mathrm{C}^{*}\left|\varepsilon_{\mathrm{MO}}\right|
$$

The coefficient of proportionality, $\mathrm{C}$, depends on the optical properties of the structure. Moreover, for a very thin Co layer embedded in a film made of continuous metallic layers, it can be shown ${ }^{[23,24]}$ that :

$$
\mathrm{C}=\mathrm{A} * d * \mathcal{E}_{p}(z) * \mathcal{E}_{s}(z)
$$

where $\mathrm{d}$ is the thickness of the Co layer, $\mathcal{E}_{p}(z)\left(\mathcal{E}_{s}(z)\right)$ is the modulus of the p-(s-) component of the EM field of a p-(s-) polarized wave incident on the continuous film structure without the Co layer calculated at the Co position, z; and A is a constant which 
depends on the optical properties of the continuous film structure without the Co layer. As an example of the validity of this thin film approximation we present in figure 4(a) (full grey line) the in-depth dependence of the EM field distribution $\mathcal{E}_{p} * \mathcal{E}_{s}$ for a $\mathrm{Cr} / \mathrm{Au}$ bilayer (2nm $\mathrm{Cr}$ and $52 \mathrm{~nm} \mathrm{Au}$ ) deposited on a glass substrate, calculated at normal incidence within the finite-difference time-domain (FDTD) formalism, using for the optical constants of $\mathrm{Au}$ and $\mathrm{Cr}$ those obtained from ellipsometry measurements on 20nm thick $\mathrm{Au}$ and $\mathrm{Cr}$ layers. The calculation was done at a wavelength of $500 \mathrm{~nm}$. As it can be observed, it is in excellent agreement with the Co dependence position of the MO activity. Also in that figure we present the in-depth dependence of the mean value of $\mathcal{E}_{p} * \mathcal{E}_{s}$ inside the Co layer calculated for a $\mathrm{Cr} / \mathrm{Au} / \mathrm{Co} / \mathrm{Au}$ layer structure (the optical properties of Co were obtained from ellipsometry measurements as mentioned above). The difference between the two theoretical curves is very small, which confirms the small perturbation induced by the Co layer on the EM distribution of the $\mathrm{Cr} / \mathrm{Au}$ structure.

The upper expression, Eq. (3), can be adapted to nanodisk structures taking into account both the variation of the p- (s- component) of the EM field within the plane of the disk and the oxidation of a thin Co layer in the lateral surface of the disks ${ }^{42}$ :

$$
|\Phi|(z) \propto \iint_{S\left(\varepsilon_{M O} \mid \neq 0\right)}\left|\varepsilon_{M O}(x, y)\right| \mathcal{E}_{s}(z, x, y) \mathcal{E}_{p}(z, x, y) d x d y .
$$

As above, in a first approximation the electromagnetic fields can be calculated considering a structure without $\mathrm{Co}$, or the Co layer can be taken into account for more generality. Figure 4(b) shows the $\mathcal{E}_{p} * \mathcal{E}_{s}$ results for both cases, obtained using the same FDTD technique. The full grey line corresponds to a $130 \mathrm{~nm}$ diameter $\mathrm{Cr} / \mathrm{Au}$ nanodisk $(2 \mathrm{~nm} \mathrm{Cr}, 52 \mathrm{~nm} \mathrm{Au}$ ) calculated at the resonance frequency. The calculation has been performed taking into account the $1 \mathrm{~nm}$ thick oxide shell surrounding the Co disk ${ }^{[42]}$, which does not contribute to the MO signal. As it can be seen, despite the simplicity of the model the U-like shape dependence of the MO activity with the Co layer position is well reproduced, although the minimum is 
located at higher values of $\mathrm{z}$ than for the experimental data. This discrepancy is due to the perturbation induced by Co in the electromagnetic field distribution. The red line represents the obtained field for similar disks, but with $6 \mathrm{~nm}$ of Co embedded in the corresponding position and $46 \mathrm{~nm} \mathrm{Au}$. The agreement with the experimental data improves, and the position of the field minima is shifted towards lower $\mathrm{z}$ values, as in the experiment.

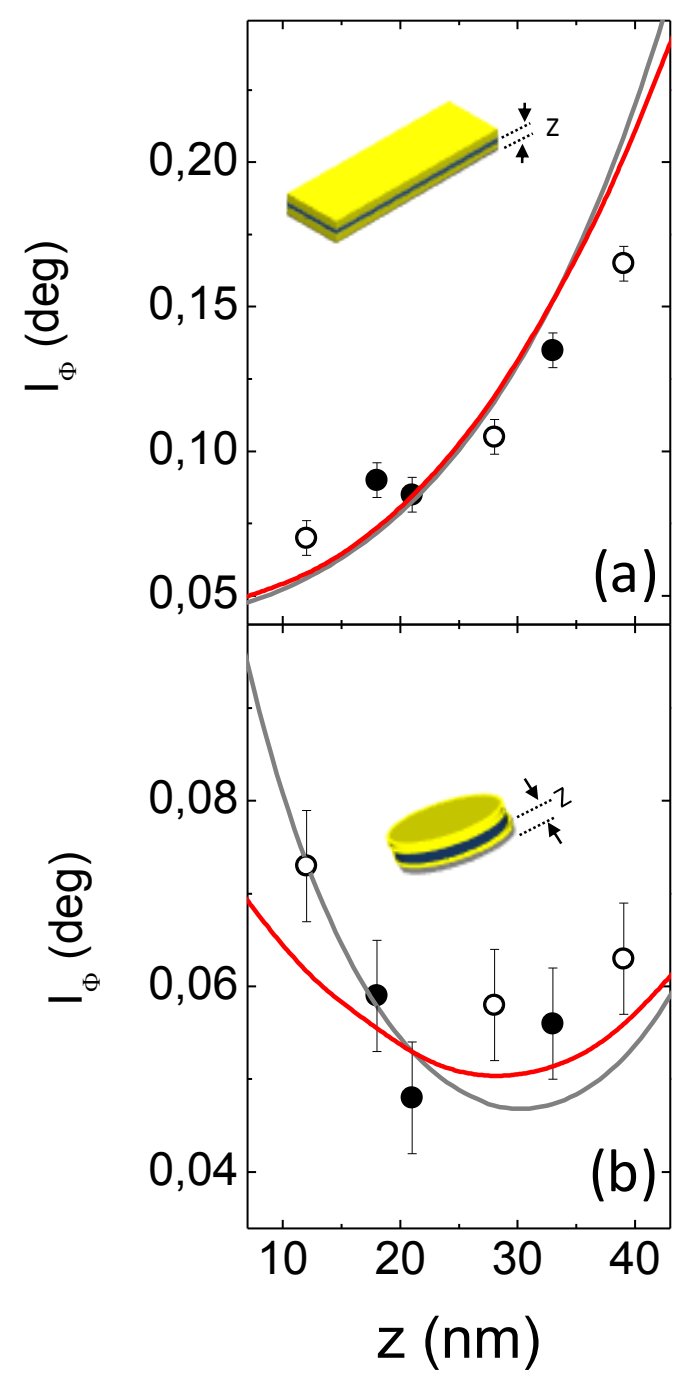

Figure 4: Intensity of the peak of the modulus of the complex Kerr rotation as a function of the Co layer position For (a) continuous films $(\lambda=500 \mathrm{~nm})$ and $(\mathrm{b})$ nanodisks samples $\left(\lambda_{\mathrm{LSPR}}\right)$. Open symbols correspond to the samples whose spectra appear in figure 3. Full lines represent theoretical calculations of the EM field distribution within the continuous film or disk structure. The grey lines correspond to the field inside a pure Au system and the red lines to the field of a system with a thin Co layer $(6 \mathrm{~nm})$ inside. 


\section{Conclusion}

In summary, we have shown that it is possible to probe the EM field distribution within a metallic nanodisk by inserting a Co layer in it and monitoring the MO activity of the system. Since this magnitude is proportional to the value of the EM field, it yields a direct measure of its value in the interior of a solid metallic entity. It is shown that the EM field exhibits maximum values near the top and bottom disk surfaces, with a minimum in between. This information could be very relevant for the design of magnetoplasmonic systems offering optimum MO enhancement, but also for other applications such as sensing or SERS, where maximum sensitivity is expected in the areas with higher EM field.

\section{Experimental Section}

Sample preparation: The colloidal lithography procedure to obtain the nanodisks samples as can be seen in the figure 5 is the following. First, polystyrene colloidal particles $(8 \% \mathrm{w} / \mathrm{v}, 100$ nm, sulfate latex, INVITROGEN) were deposited by electrostatic self-assembly onto glass substrate precoated with $\sim 360 \mathrm{~nm}$ of PMMA A4 (1). The surface of the PMMA A4 was treated with an oxygen plasma (50W, 450 mTorr) to make it hydrophilic (2), and then a single layer precursor film was absorbed to make the surface positively charged using $0.02 \%$ (by weight) poly(diallyldimethylammonium chloride) (PDDA, MW 400 000-/500 000, Sigma Aldrich) (3), rinsing in water during $60 \mathrm{~s}$ and finally drying with nitrogen gas. Negatively charged polystyrene particles are adsorbed onto the charged substrates from solution by electrostatic interactions. Particle concentrations of $0.02 \%$ (by weight) were used. Adsorption time was 1 min to allow the adsorption to reach saturation and a better uniform distribution in all experiments (4). Excess particles were rinsed off under running water and the samples were blown dry with nitrogen gas. Milli-Q water (Millipore) was used at all times. The density of spheres was selected to obtain a disk concentration of $12 \%$. After this, Au thin films $(15 \mathrm{~nm})$ were deposited by sputtering (5). Oxygen plasma treatment (50W, $450 \mathrm{mTorr})$ 
for $15 \mathrm{~s}$ was used again to clean the Au surface. Polystyrene particles were retired of the surface substrate using tape striping (6). Then, the sample was exposed in an oxygen plasma treatment (50 W, 7.5x10-9 Torr) to attack the PMMA and drill the holes (7). Subsequently, the substrates were introduced in a UHV deposition system (8) where, after deposition of $2 \mathrm{~nm}$ $\mathrm{Cr}$ to improve the adhesion of the disks to the substrate, $\mathrm{a} \mathrm{Au} / \mathrm{Co} / \mathrm{Au}$ trilayer was grown with a fixed Co thickness of about $6 \mathrm{~nm}$ and a total Au thickness fixed to $46 \mathrm{~nm}$ approximately. $\mathrm{Cr}$ and Co were deposited by magnetron sputtering (1.5x10-3 mbar; $0.23 \AA / \mathrm{sec}$ and $1.5 \times 10-3$ mbar; $0.15 \AA / \mathrm{sec}$, Ar pressure and deposition rates respectively) and Au by thermal evaporation (0.25 $\AA / \mathrm{sec}$ deposition rate). After lift-off (9), an ensemble of nanodisks randomly oriented on the glass substrate were obtained.

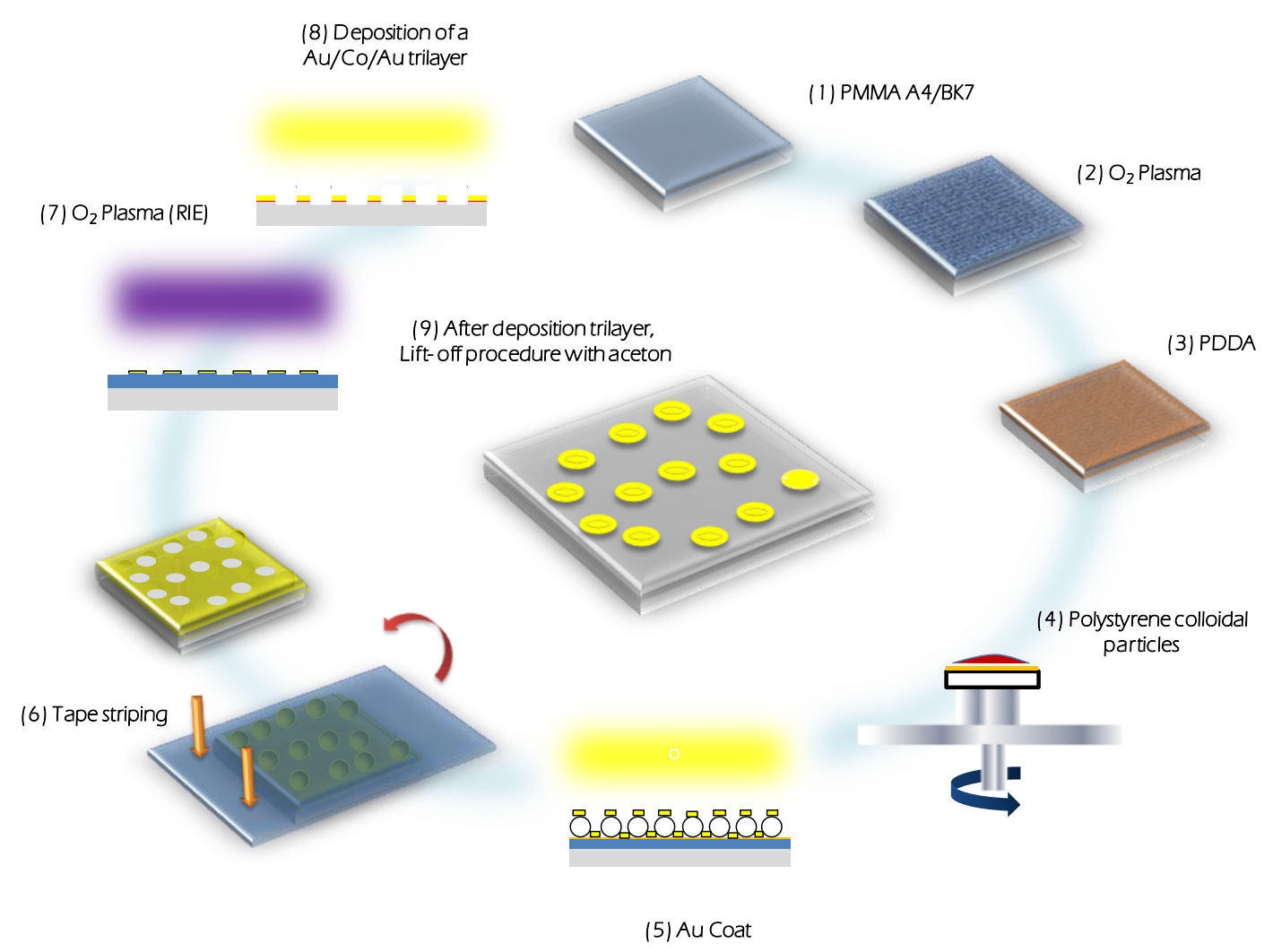

Figure 5. Process of fabrication of the metallic nanodisk by colloidal lithography.

Kerr spectroscopy: The MO characterization of the samples has been carried out by means of Kerr spectroscopy in the polar configuration. The sample, placed inside an electromagnet 
that applies a magnetic field perpendicular to its surface, is illuminated at normal incidence by a monochromatic beam coming from a Xe lamp followed by a monochromator. By modulating the beam polarization with a photoelastic modulator, the Kerr rotation and ellipticity for each wavelength are determined. The measurements have been performed with an applied magnetic field, which insures magnetic saturation of the structures.

\section{Acknowledgements}

The authors acknowledge funding support from the EU (NMP3-SL-2008-214107Nanomagma), the Spanish MICINN ("FUNCOAT" CONSOLIDER INGENIO 2010 CSD2008-00023 and MAGPLAS MAT2008-06765-C02-01/NAN), the Comunidad de Madrid ("NANOBIOMAGNET", S2009/MAT - 1726 and "MICROSERES-CM", S2009/ TIC-1476) and CSIC (JAE fellowship for E. Ferreiro-Vila and JAE Doc fellowship for D. Meneses-Rodríguez).

\section{References}

[1] S. A Maier Plasmonics: Fundamentals and Applications, Ed. Springer, Berlin, 2007.

[2] S. A. Maier, P. G. Kik, H. A. Atwater, S. Meltzer, E. Harel, B. E. Koel and A. G Requicha, Nature Mater. 2003, 2, 229.

[3] A. L. Pyayt, B. Wiley, Y. Xia, A. Chen and L. Dalton, Nat. Nanotechnol. 2008, 3, 660.

[4] T. H. Taminiau, F. D. Stefani, F. B. Segerink, N. F. Van Hulst, Nat Photon 2008, 2, 234-237.

[5] E. Betzig, J. Trautman, T. Harris, Weiner J. and R. Kostelak, Science 1991, 251, 1468.

[6] S. Palomba, L. Novotny, Nano Lett. $\quad$ 2009, 9, 3801.

[7] V. Krachmalnicoff, E. Castanié, Y. De Wilde and R. Carminati, Phys. Rev. Lett. 2010, $105,183901$. 
[8] M. A. Noginov, G. Zhu, A. M. Belgrave, R. Bakker, V. M. Shalaev, E. E. Narimano, S. Stout, E. Herz, T. Suteewong and U. Wiesner, Nature 2009, 460, 1110.

[9] R. F. Oulton, V. J. Sorger, T. Zentgraf, R.-M. Ma, C. Gladden, L. Dai, G. Bartal and X. Zhang, Nature 2009, 461, 629.

[10] S. Nie and S. R. Emory, Science 1997, 275, 1102.

[11] H. X. Xu, E. J. Bjerneld M. , Käll, L. Börjesson, Phys. Rev. Lett. 1999, 83, 4357-4360.

[12] A. J. Haes, R. P. A Van Duyne, J. Am. Chem. Soc. 2002, 124,10596.

[13] N. Nath, A. Chilkoti, Anal. Chem. 2004, 76, 5370.

[14] T. Endo, K. Kerman, N. Nagatani, Y. Takamura, E. Tamiya, Anal. Chem. 2005, 77, 6976-6984.

[15] K. L. Kelly, E. Coronado, L. L. Zhao, G. C. Schatz, J. Phys. Chem. B 2003, 107,-677.

[16] P. Muhlschlegel, H.-J. Eisler, O. J. F. Martin, B. Hecht and D. W. Pohl, Science 2005, $308,1607$.

[17] H. Okamoto and K. Imura, Progress in Surface Science 2009, 84, 199.

[18] M. Schnell, A. Garcia-Etxarri, A. J. Huber, K. Crozier, J. Aizpurua, R. Hillenbrand, Nat Photon 2009, 3, 287.

[19] J. Nelayah, M. Kociak, O. Stephan, F. J. García de Abajo, M. Tence, L. Henrard, D. Taverna, I. Pastoriza-Santos, L. M. Liz-Marzan; C. Colliex, Nat. Phys. 2007, 3, 348.

[20] L. Douillard, F. Charra, Z. Korczak, R. Bachelot, S. Kostcheev, G. Lerondel, P.-M. Adam, P. Royer, Nano Lett. 2008, 8, 935.

[21] V. V. Temnov, G. Armelles, U. Woggon, D. Guzatov, A. Cebollada, A. Garcia-Martin, J. M. Garcia-Martin, T. Thomay, A. Leitenstorfer, R. Bratschitsch, Nat. Photonics 2010, 4, 107. 
[22] D. Martín-Becerra, J. B. González-Díaz, V. V. Temnov, A. Cebollada, G. Armelles, T. Thomay, A. Leitenstorfer, R. Bratschitsch, A. García-Martín and M. U. González, Appl. Phys. Lett. 2010, 97, 183114.

[23] P. Bertrand, C. Hermann, G. Lampel, J. Peretti and V.I. Safarov, Phys. Rev B 2001, 64, 235421.

[24] S. Visnovsky, M. Nyvlt, V. Prosser, R. Lopusnik, R. Urban, J. Ferre, G. Penissard, D Renard. and R. Krishnan, Phys. Rev B 1995, 52, 1090.

[25] V. I. Belotelov, L. L. Doskolovich and A. K. Zvezdin, Phys. Rev. Lett. 2007, 98, 077401.

[26] C. Clavero, K. Yang, J.R. Skuza and R.A. Lukaszew, Opt. Express 2010, 18, 7743.

[27] G. Armelles, J. B. González-Díaz, A. García-Martín, J. M. García-Martín, A. Cebollada, M. U. González, S. Acimovic, J. Cesario, R. Quidant, G. Badenes, Opt. Express 2008, 16, 16104-16112.

[28] L. Wang, C. Clavero, Z. Huba, K. J. Carroll, E. E. Carpenter, D. Gu and R. A.Lukaszew, Nano Lett. 2011, 11, 1237.

[29] P. Hanarp, D. S. Sutherland, J. Gold and B. Kasemo, Colloids Surf. A 2003, 214, 23

[30] J.C. Hulteen and R.P. Van Duyne, J. Vac. Sci. Technol. A, 1995,13, 1553-8.

[31] P. Hanarp, M. Käll and D. S. Sutherland, J. Phys. Chem. B, 2003, 107, 5768.

[32] F. A. Denis, P. Hanarp, D. S Sutherland and Y. F. Dufrene, Nano Lett. 2002, 2, 1419.

[33] W. S. Kim, M. Aderholz W. and Kleemann, Meas. Sci. Technol. 1993, 4, 1275.

[34] J. B. González-Díaz, A. García-Martín, J. M. García-Martín, A. Cebollada, G. Armelles, B. Sepúlveda Y. Alaverdyan and M. Käll, Small 2008, 4, 202.

[35] P. K. Jain, Y. Xiao, R. Walsworth, A. E. Cohen, Nanocrystals. Nano Lett. 2009, 9, 1644. 
[36] G. X. Du, T. Mori, M. Suzuki, S. Saito, H. Fukuda, M. Takahashi, Appl. Phys. Lett. 2010, 96, 081915.

[37] B. Sepúlveda, J. B. González-Díaz, A. García-Martín, L. M. Lechuga and G. Armelles, Phys. Rev. Lett. 2010,104, 147401.

[38] J. B. González-Díaz, B. Sepúlveda, A. García-Martín, G. Armelles, Appl. Phys. Lett. 2010, $97,043114$.

[39] G. Armelles, A. Cebollada, A. Garcia-Martin, J. M. Garcia-Martin, M. U. Gonzalez, J. B. Gonzalez-Diaz; E. Ferreiro-Vila, J. F. Torrado, J. Opt. A: Pure Appl. Opt. 2009, $11,114023$.

[40] G. X. Du, T. Mori, M. Suzuki, S. Saito, H. Fukuda, M. Takahashi, J. Appl. Phys. 2010, 107, 09A928.

[41] L. Wang, K. Yang, C. Clavero, A. J. Nelson, K. J. Carroll, E. E. Carpenter, R. A. Lukaszew, Nanoparticles. J. Appl. Phys. 2010, 107, 09B303.

[42] L. Balcells, B. Martínez, O. Iglesias, J. M. García-Martín, A. Cebollada, A. GarcíaMartín, G. Armelles, B. Sepúlveda and Y. Alaverdyan, Phys. Lett. 2009, 94, 062502. 